\title{
On the Simulation and Correlation Properties of Phase-Envelope Nakagami Fading Processes
}

\author{
José Cândido Silveira Santos Filho and Michel Daoud Yacoub
}

\begin{abstract}
We propose a general method for simulating both envelope and phase of Nakagami fading channels, allowing for arbitrary values of fading parameter and nonisotropic scenarios. The method complies with the newly-derived Nakagami phaseenvelope distribution and Nakagami envelope autocorrelation function. In passing, we also address the unsettled problem of how to autocorrelate Nakagami phase samples appropriately. In the proposed scheme, Nakagami samples are first drawn and then suitably arranged to match the correlation properties of the channel. The arrangement follows the rank statistics of a Rayleigh (complex Gaussian) reference sequence with the desired autocorrelation. Relying on this scheme, we also derive simple, highly-precise, closed-form approximations to the envelope autocorrelation and to the unknown phase autocorrelation, quadrature component autocorrelation, and quadrature component crosscorrelation of the Nakagami channel. Sample results are presented, and practically no distinction is observed between the derived approximations and the simulation points.
\end{abstract}

Keywords-Correlation, fading channels, Nakagami fading, rank statistics, simulation.

\section{INTRODUCTION}

W HEN proposed, the Nakagami fading model specified the distribution of the channel envelope but not of the channel phase [1]. Because of this, and knowing that the phase distribution of the Rayleigh channel-a special case of Nakagami fading-is uniform, researchers have arbitrarily assigned a uniform distribution to the Nakagami phase [2]. Recently, a simple phase-envelope model was proposed for Nakagami fading which accounts for the effects of the fading parameter on both the phase and the envelope of the channel [3]. In this model, the channel envelope remains the wellknown Nakagami envelope, but the channel phase is no longer uniform, except for the special Rayleigh case. The primary motivation for the use of the Nakagami phase-envelope model proposed in [3] is that its phase distribution coincides with those of Rice and Hoyt for the limiting cases in which their envelope distributions are known to coincide. Furthermore, the proposed Nakagami phase distribution bears similar shapes to those of Rice and Hoyt for the remaining cases, in which their envelope distributions approach each other. In contrast, the usual assumption of uniform phase for Nakagami fading does not comply with these features.

Despite the surge of research in the last decades, it remains unclear what some Nakagami high-order statistics should be. On the one hand, there exists a well-established realization model for the channel envelope: the square root of the sum

The authors are with the Department of Communications, School of Electrical and Computer Engineering, University of Campinas, PO Box 6101, 13083-852 Campinas, SP, Brazil (Tel: +55 (19) 3788 5106, Fax: +55 (19) 3289 1395, E-mail:\{candido,michel\}@wisstek.org). of independent squared Rayleigh envelopes [1], [4]. Based on this model, the envelope second-order distribution and autocorrelation function can be derived as in [1, Eq. (126)] and [5, Eq. (1)], respectively. On the other hand, no realization model has been established yet for the Nakagami phase, neither by physical arguments nor by empirical evidences. As a result, the phase second-order distribution and autocorrelation function are unknown.

Because of the longstanding uncertainties regarding the phase and the high-order statistics of Nakagami fading, most simulators have used particular assumptions in order to implement the phase and the autocorrelation of the channel (cf. [2] and references therein). In this work, we propose a general method for simulating both the envelope and the phase of Nakagami fading channels, allowing for arbitrary values of fading parameter and nonisotropic scenarios. The method complies with the newly-derived Nakagami phase-envelope distribution [3] and Nakagami envelope autocorrelation function [5]. In passing, we also address the unsettled problem of how to autocorrelate Nakagami phase samples appropriately. In the proposed scheme, Nakagami samples are first drawn and then suitably arranged to match the correlation properties of the channel. The arrangement follows the rank statistics of a Rayleigh (complex Gaussian) reference sequence with the desired autocorrelation. Relying on this scheme, we also derive simple, highly-precise, closed-form approximations to the envelope autocorrelation and to the unknown phase autocorrelation, quadrature component autocorrelation, and quadrature component crosscorrelation of the Nakagami channel. Sample results are presented, and practically no distinction is observed between the derived approximations and the simulation points.

\section{NAKAgami Model}

Let the complex-valued Nakagami fading channel $Z$ be represented as

$$
Z=X+j Y=R \exp (j \Theta),
$$

where $X, Y, R$, and $\Theta$ are the in-phase component, the quadrature component, the envelope, and the phase, respectively. In this section, we revisit some statistics of $Z$.

The probability density function (PDF) of the Nakagami fading envelope $R$ is given by [1]

$$
f_{R}(r)=\frac{2 m^{m} r^{2 m-1}}{\Gamma(m) \Omega^{m}} \exp \left(-\frac{m r^{2}}{\Omega}\right), 0 \leq r<\infty,
$$

where $\Omega=E\left[R^{2}\right]$ is the mean power, $m=\Omega^{2} / V\left[R^{2}\right]$ is the Nakagami fading parameter, and $\Gamma(\cdot)$ is the gamma function. $(E[\cdot]$ denotes expectation, $V[\cdot]$ variance.) Based on physical 


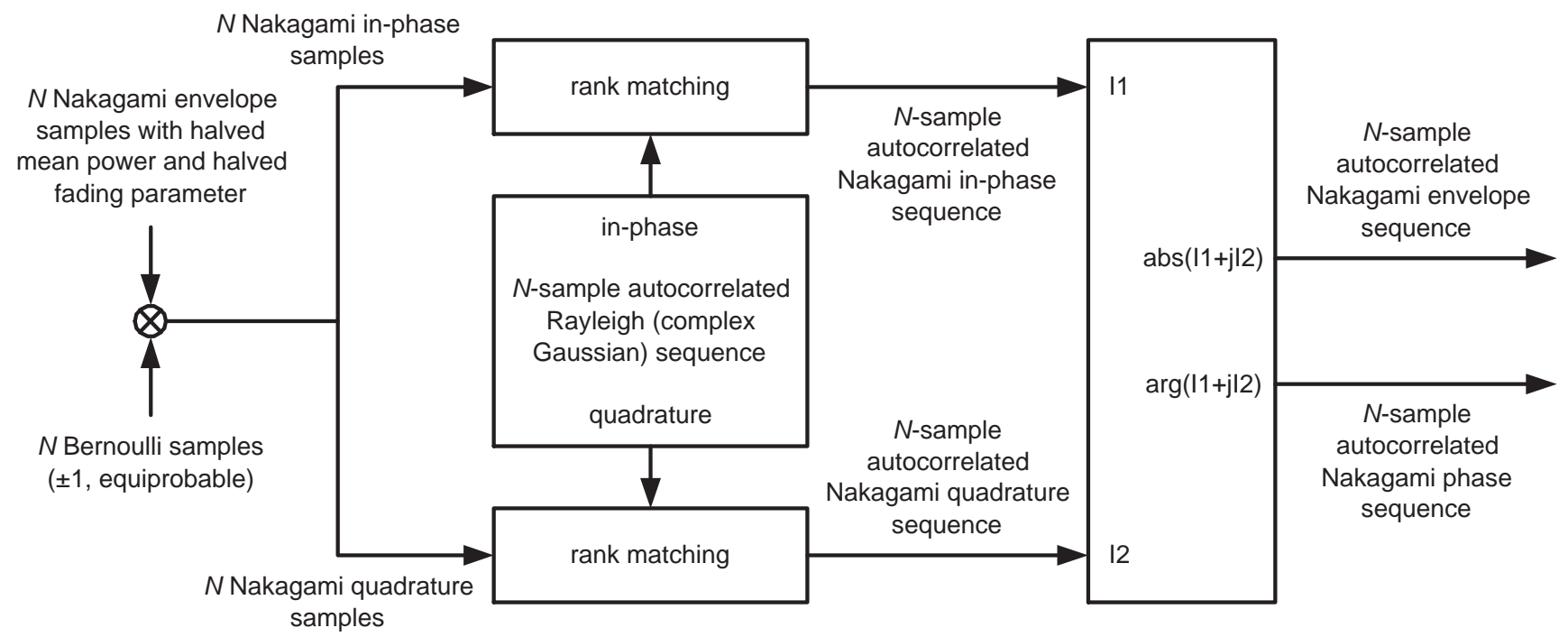

Fig. 1. The Nakagami fading channel simulator.

arguments, the PDFs of $\Theta, X$, and $Y$ have been recently derived in [3] as

$$
\begin{gathered}
f_{\Theta}(\theta)=\frac{\Gamma(m)|\sin 2 \theta|^{m-1}}{2^{m} \Gamma^{2}\left(\frac{m}{2}\right)},-\pi \leq \theta<\pi \\
f_{X}(u)=f_{Y}(u)=\frac{m^{\frac{m}{2}}|u|^{m-1}}{\Omega^{\frac{m}{2}} \Gamma\left(\frac{m}{2}\right)} \exp \left(-\frac{m u^{2}}{\Omega}\right),-\infty<u<\infty .
\end{gathered}
$$

For $m=1$, (3) and (4) specialize to the uniform phase PDF and Gaussian quadrature component PDF of the Rayleigh channel, respectively. However, for $m \neq 1$, the Nakagami phase is non-uniform and the Nakagami quadrature components are non-Gaussian.

The autocorrelation function (ACF) of the Nakagami envelope has been derived in [5] based on the physical model of the squared Nakagami envelope as the sum of $m$ i.i.d. squared Rayleigh envelopes [1], [4]. The Nakagami envelope $\mathrm{ACF}$ is [5]

$$
A_{R}(\tau)=\frac{\Omega \Gamma^{2}\left(m+\frac{1}{2}\right)}{m \Gamma^{2}(m)}{ }_{2} F_{1}\left(-\frac{1}{2},-\frac{1}{2} ; m ; \rho_{R^{2}}^{\mathrm{ray}}(\tau)\right)
$$

where $\tau$ is a time lag, ${ }_{2} F_{1}(\cdot, \cdot ; \cdot ; \cdot \cdot)$ is the hypergeometric function, and $\rho_{R^{2}}^{\text {ray }}(\tau)$ is the autocorrelation coefficient (ACC) of each underlying squared Rayleigh envelope process. The statistical properties of Rayleigh (complex Gaussian) fading processes and expressions for $\rho_{R^{2}}^{\text {ray }}(\tau)$ have been extensively reported in the literature for a myriad of fading scenarios (e.g. [6] and [7]). In contrast, to the best of the authors' knowledge, no expressions have been established yet for the phase and quadrature component ACFs of Nakagami fading channels.

\section{NAKAGAMI SimUlator}

Our aim is to generate an $N$-sample ( $N$ being an arbitrary positive integer) sequence for $Z$ that matches (4)—or, equivalently, (2) and (3)-and (5), for any desired values of $\Omega$, $m$, and $\rho_{R^{2}}^{\text {ray }}(\tau)$. The problem is that no realization model for $Z$ exists, except for its envelope, whose square value equals the sum of $m$ i.i.d. squared Rayleigh envelopes [1], [4], [5]. Furthermore, the use of the envelope model as a simulation scheme is limited to integer values of $m$.

We circumvent the above problem by choosing a different simulation approach: (i) draw first $N$ samples following the distribution of $Z$ and (ii) then suitably arrange these samples to match the desired autocorrelation properties. Step (i) complies with the static properties (distribution) of the process, described in (4) or, equivalently, in (2) and (3); step (ii) complies with the dynamic properties (autocorrelation), described in (5). This approach was introduced in [8] for simulating the Nakagami envelope process. Here, we shall extend it to the phase-envelope simulation.

Step (i) is simple. In order to address it, one may decompose the complex $Z$ samples in terms of $(X, Y)$ or $(R, \Theta)$; we choose $(X, Y)$. Note in (4) that $X$ and $Y$ are identically distributed. Thus, a unique set of $N$ samples matching (4) can be used to compose both the $X$ and $Y$ sequences. As shown in [3], the modulus of $X$ and $Y$ follows a Nakagami envelope distribution with halved mean power $\Omega / 2$ and halved fading parameter $m / 2$. In addition, positive and negative values of $X$ and $Y$ occur with identical probability [3]. Therefore, the samples for $X$ and $Y$ can be obtained from the product of Nakagami envelope samples (with halved mean power $\Omega / 2$ and halved fading parameter $m / 2$ ) and Bernoulli samples ( \pm 1 , equiprobable), as sketched in the leftmost side of Fig. 1 . Routines for generating Nakagami envelope samples (root squared gamma) and Bernoulli samples are available in most standard computing packages such as MATHEMATICA and MATLAB.

In contrast, step (ii) is intricate. One out of $N ! \times N !$ inphase and quadrature sample arrangements must be found that render a good fit to the analytical envelope ACF in (5). 
Of course, the brute-force approach-comparing all possible arrangements-is not practicable. Instead, we shall use an insightful and fundamental result from [8]: the rank statistics of the Nakagami envelope are closely approximated by those of each underlying Rayleigh envelope, irrespective of the fading parameter $m$. This has been used in [8] to generate Nakagami envelope sequences that match the analytical envelope ACF, by arranging the Nakagami envelope samples according to the rank statistics of a Rayleigh envelope reference sequence with the desired autocorrelation $\rho_{R^{2}}^{\text {ray }}(\tau)$. The operation was called rank matching and can be easily accomplished through builtin routines of standard computing packages [8]. In short, if an $N$-sample sequence $A$ is rank-matched to an $N$-sample sequence $B$, then each sample in $A$ is arranged in the same position of the sample in $B$ with the same statistical $\operatorname{rank}^{1}$ of that sample in $A$.

Here we have to arrange in-phase and quadrature samples instead of just envelope samples. Probably there exist several ways to arrange the in-phase and quadrature samples so that the resulting envelope sequence fits the analytical envelope ACF. However, one useful requisite is to maintain the solution as simple as possible. Then, knowing from [8] that the resulting Nakagami envelope sequence should approach the rank statistics of a Rayleigh envelope sequence, a simple solution is to rank-match the Nakagami in-phase sequence to a Rayleigh in-phase sequence, and the Nakagami quadrature sequence to a Rayleigh quadrature sequence. The simulation scheme is shown in Fig. 1. As for the Rayleigh (complex Gaussian) reference sequence with the desired autocorrelation, it can be generated by any method available in the literature. Note that both isotropic as well as nonisotropic Rayleigh reference sequences can be used, leading to isotropic or nonisotropic Nakagami sequences correspondingly.

A last remark is noteworthy. Although in the above discussion we have not explicitly addressed the Nakagami phase autocorrelation (which is indeed unknown), the proposed scheme embeds an assumption on this issue, as follows. By choosing to rank-match both the in-phase and quadrature components of the Nakagami sequence to the corresponding components of a Rayleigh sequence, we are equivalently rank-matching not only the resulting Nakagami envelope sequence to a Rayleigh envelope sequence but also the resulting Nakagami phase sequence to a Rayleigh phase sequence. On the one hand, the envelope rank-matching is desired, since this is known to comply with the realization model for the Nakagami envelope and its associated envelope ACF [8]. On the other, the phase rank-matching is indeed an arbitrary assumption, since no realization model for the Nakagami phase has been established yet, rendering the phase ACF unknown. In other words, it was shown in [8] that the rank statistics of the Nakagami envelope approach those of the Rayleigh envelope; here, for simplicity, we reproduce the envelope behavior in the phase by assuming that the rank statistics of the Nakagami phase approach those of the Rayleigh phase. Although this assumption is somewhat arbitrary, it renders the Nakagami correlation properties at-

\footnotetext{
${ }^{1}$ Statistical rank is the ordinal number of a value in a list arranged in a specified (decreasing or increasing) order.
}

tractive, for these can be closely approximated in terms of the well-known correlation properties of the Rayleigh channel, as detailed next.

\section{Output Statistics}

The simulation method proposed here inherently complies with the exact PDFs (phase, envelope, and quadrature components) of the Nakagami fading model and, as shall be seen from sample examples, precisely approximates the analytical Nakagami envelope ACF. As for the phase and quadrature component ACFs, although not established a priori, these can be now examined based on the designed simulation scheme itself. In the following, we derive simple, precise, closed-form approximations to these statistics.

For any stationary random process, the ACC of the samples is known to be approximated by the ACC of their statistical ranks [9]. Then, by rank-matching the Nakagami sequence to a Rayleigh sequence, we indeed produce a Nakagami sequence whose ACC approximates that of the Rayleigh sequence. This is a central result: in the proposed simulation scheme, for any value of the fading parameter $m$, the ACC of a given Nakagami component-e.g. the phase, the envelope, the quadrature component or any function of them-approximates the ACC of the corresponding component in the reference Rayleigh process. Accordingly, denoting by $\rho_{R}^{\operatorname{nak}}(\tau), \rho_{\Theta}^{\text {nak }}(\tau), \rho_{X}^{\operatorname{nak}}(\tau)$, and $\rho_{Y}^{\operatorname{nak}}(\tau)$ the envelope, phase, in-phase component, and quadrature component ACCs of the Nakagami channel, and by $\rho_{R}^{\text {ray }}(\tau), \rho_{\Theta}^{\text {ray }}(\tau), \rho_{X}^{\text {ray }}(\tau)$, and $\rho_{Y}^{\text {ray }}(\tau)$ the corresponding ACCs of the Rayleigh channel, it follows that for any value of $m$

$$
\begin{aligned}
\rho_{R}^{\text {nak }}(\tau) & \approx \rho_{R}^{\mathrm{ray}}(\tau) \\
\rho_{\Theta}^{\mathrm{nak}}(\tau) & \approx \rho_{\Theta}^{\mathrm{ray}}(\tau) \\
\rho_{X}^{\mathrm{nak}}(\tau)=\rho_{Y}^{\mathrm{nak}}(\tau) & \approx \rho_{X}^{\mathrm{ray}}(\tau)=\rho_{Y}^{\mathrm{ray}}(\tau) .
\end{aligned}
$$

The same rationale can be applied to crosscorrelation coefficients (CCCs). For instance, the CCC $\rho_{X, Y}^{\text {nak }}(\tau)$ between the Nakagami in-phase and quadrature components can be approximated by the Rayleigh one, i.e.

$$
\rho_{X, Y}^{\text {nak }}(\tau) \approx \rho_{X, Y}^{\text {ray }}(\tau) .
$$

Similar results can be formulated for auto- and crosscorrelations involving any function of the fading components $X, Y$, $R$, and $\Theta$.

From the above approximations and using the standard definition for auto- and crosscorrelation coefficients, corresponding approximations to the auto- and crosscorrelation functions of the Nakagami fading components can be derived as

$$
\begin{gathered}
A_{R}(\tau) \approx \rho_{R}^{\mathrm{ray}}(\tau) E\left[R^{2}\right]+\left(1-\rho_{R}^{\mathrm{ray}}(\tau)\right) E^{2}[R] \\
A_{\Theta}(\tau) \approx \rho_{\Theta}^{\mathrm{ray}}(\tau) E\left[\Theta^{2}\right]+\left(1-\rho_{\Theta}^{\mathrm{ray}}(\tau)\right) E^{2}[\Theta] \\
A_{X}(\tau)=A_{Y}(\tau) \approx \rho_{X}^{\mathrm{ray}}(\tau) E\left[X^{2}\right]+\left(1-\rho_{X}^{\mathrm{ray}}(\tau)\right) E^{2}[X] \\
A_{X, Y}(\tau) \approx \rho_{X, Y}^{\text {ray }}(\tau) E[X Y]+\left(1-\rho_{X, Y}^{\text {ray }}(\tau)\right) E[X] E[Y]
\end{gathered}
$$


Finally, assuming that $E\left[R^{2}\right], E\left[\Theta^{2}\right], E\left[X^{2}\right], E\left[Y^{2}\right]$, and $E[X Y]$ are unity, we obtain the normalized auto- and crosscorrelation functions as

$$
\begin{gathered}
\tilde{A}_{R}(\tau) \approx \rho_{R}^{\mathrm{ray}}(\tau)+\left(1-\rho_{R}^{\mathrm{ray}}(\tau)\right) \frac{\Gamma^{2}\left(m+\frac{1}{2}\right)}{m \Gamma^{2}(m)} \\
\tilde{A}_{\Theta}(\tau) \approx \rho_{\Theta}^{\mathrm{ray}}(\tau) \\
\tilde{A}_{X}(\tau)=\tilde{A}_{Y}(\tau) \approx \rho_{X}^{\mathrm{ray}}(\tau) \\
\tilde{A}_{X, Y}(\tau) \approx \rho_{X, Y}^{\text {ray }}(\tau) .
\end{gathered}
$$

In (9), we have used $E[\Theta]=E[X]=E[Y]=0$ and $E[R]=(\Gamma(m+1 / 2) /(\Gamma(m) \sqrt{m})) E\left[R^{2}\right]$. Note that only the approximate normalized envelope ACF depends on the fading parameter $m$.

The approximations derived in (6)-(9) for Nakagami fading rely on expressions for the correlation properties of Rayleigh fading processes: $\rho_{R}^{\text {ray }}(\tau), \rho_{\Theta}^{\text {ray }}(\tau), \rho_{X}^{\text {ray }}(\tau), \rho_{Y}^{\text {ray }}(\tau)$, and $\rho_{X, Y}^{\text {ray }}(\tau)$. These expressions are extensively reported in the literature for a myriad of fading scenarios (e.g. [6] and [7]). A sample case is presented next.

\section{Simulation Results}

In this section, sample simulation results are presented for the general case of nonisotropic fading scenarios. In such scenarios, the distribution of the angle of arrival (AOA) of the multipath waves is nonuniform. A plausible model for the directional AOA is the parametric Von Mises/Tikhonov distribution, for which the Rayleigh quadrature component ACC and CCC are given respectively by [7]

$$
\begin{aligned}
& \rho_{X}^{\text {ray }}(\tau)=\operatorname{Re}\left[\frac{I_{0}\left(\sqrt{\kappa^{2}-\left(2 \pi f_{D} \tau\right)^{2}+j 4 \kappa \pi f_{D} \tau \cos \mu}\right)}{I_{0}(\kappa)}\right] \\
& \rho_{X, Y}^{\text {ray }}(\tau)=\operatorname{Im}\left[\frac{I_{0}\left(\sqrt{\kappa^{2}-\left(2 \pi f_{D} \tau\right)^{2}+j 4 \kappa \pi f_{D} \tau \cos \mu}\right)}{I_{0}(\kappa)}\right],
\end{aligned}
$$

where $\operatorname{Re}[\cdot]$ denotes real part, $\operatorname{Im}[\cdot]$ denotes imaginary part, $I_{0}(\cdot)$ is the modified Bessel function of the first kind and zeroth order, $f_{D}$ is the maximum Doppler frequency in $\mathrm{Hz}$, $\mu$ represents the mean direction of the AOA, and $\kappa$ controls the beamwidth. In particular, for $\kappa=0$, we have the isotropic scenario with uniform AOA.

Following [6], $\rho_{R}^{\text {ray }}(\tau)$ and $\rho_{\Theta}^{\text {ray }}(\tau)$ can be obtained from $\rho_{X}^{\text {ray }}(\tau)$ and $\rho_{X, Y}^{\text {ray }}(\tau)$ as

$$
\begin{gathered}
\rho_{R}^{\text {ray }}(\tau)=\frac{1-{ }_{2} F_{1}\left(-\frac{1}{2},-\frac{1}{2} ; 1 ; \lambda^{2}(\tau)\right)}{1-\frac{4}{\pi}} \\
\rho_{\Theta}^{\text {ray }}(\tau)=3 f_{1}(\tau)\left(1+2 f_{1}(\tau)\right)-\frac{1}{8} f_{2}(\tau),
\end{gathered}
$$

where

$$
\begin{gathered}
\lambda(\tau) \triangleq\left|\rho_{X}^{\text {ray }}(\tau)+j \rho_{X, Y}^{\text {ray }}(\tau)\right| \\
\phi(\tau) \triangleq \arg \left(\rho_{X}^{\text {ray }}(\tau)+j \rho_{X, Y}^{\text {ray }}(\tau)\right) \\
f_{1}(\tau) \triangleq \frac{1}{2 \pi} \arcsin (\lambda(\tau) \cos \phi(\tau))
\end{gathered}
$$

$$
f_{2}(\tau) \triangleq \frac{6 \operatorname{Li}_{2}\left(\lambda^{2}(\tau)\right)}{\pi^{2}}
$$

in which $\mathrm{Li}_{2}(\cdot)$ is the dilogarithm function. Finally, the squared Rayleigh envelope ACC $\rho_{R^{2}}^{\text {ray }}(\tau)$ is given by [6]

$\rho_{R^{2}}^{\text {ray }}(\tau)=\lambda^{2}(\tau)=\left|\frac{I_{0}\left(\sqrt{\kappa^{2}-\left(2 \pi f_{D} \tau\right)^{2}+j 4 \kappa \pi f_{D} \tau \cos \mu}\right)}{I_{0}(\kappa)}\right|^{2}$.

Following the Von Mises/Tikhonov model, Figs. 2 and 3 present the approximate and simulated normalized Nakagami auto- and crosscorrelation functions for isotropic $(\kappa=0)$ and nonisotropic $(\kappa=1)$ scenarios, respectively. For the channel envelope, the exact analytical ACF is also shown. The required Rayleigh reference sequences were generated using the autoregressive method in [10]. In the examples, $\mu=0$, $N=2^{20}$, and the fading parameter $m$ assumes different values. Note, in all of the cases, the excellent match to the exact envelope autocorrelation, and how the approximations to the envelope, phase, and quadrature autocorrelations and to the quadrature crosscorrelation are highly precise and practically indistinguishable from the simulation points. Also note that, apart from the envelope autocorrelation, the remaining correlation functions are hardly dependent on the fading parameter $m$, as predicted in the derived approximations. Comparisons involving first-order statistics have been omitted, for the proposed scheme inherently complies with them.

\section{CONCLUSION}

A general simulation method was proposed for Nakagami fading channels, allowing for arbitrary values of fading parameter and nonisotropic scenarios, and matching the Nakagami phase-envelope distribution and envelope autocorrelation function. We also derived simple, highly-precise, closedform approximations to the envelope autocorrelation and to the unknown phase autocorrelation, quadrature component autocorrelation, and quadrature component crosscorrelation of the Nakagami channel.

\section{REFERENCES}

[1] M. Nakagami, "The $m$-distribution - a general formula of intensity distribution of rapid fading," in Statistical Methods in Radio Wave Propagation, W. C. Hoffman, Ed. Oxford, England: Pergamon, 1960.

[2] N. C. Beaulieu and C. Cheng, "Efficient Nakagami-m fading channel simulation," IEEE Trans. Veh. Technol., vol. 54, no. 2, pp. 413-424, Mar. 2005.

[3] M. D. Yacoub, G. Fraidenraich, and J. C. S. Santos Filho, "Nakagami$m$ phase-envelope joint distribution," Electron. Lett., vol. 41, no. 5, pp. 259-261, Mar. 2005.

[4] M. D. Yacoub, J. E. V. Bautista, and L. G. de Resende Guedes, "On higher order statistics of the Nakagami- $m$ distribution," IEEE Trans. Veh. Technol., vol. 48, no. 3, pp. 790-794, May 1999.

[5] J. C. S. Santos Filho, G. Fraidenraich, U. S. Dias, and M. D. Yacoub, "On the Nakagami- $m$ crosscorrelation function," in Proc. IMOC, Brasília, Brazil, 25-28 July 2005.

[6] W. C. Jakes, Microwave Mobile Communications. New York: Wiley, 1974.

[7] A. Abdi, J. A. Barger, and M. Kaveh, "A parametric model for the distribution of the angle of arrival and the associated correlation function and power spectrum at the mobile station," IEEE Trans. Veh. Technol., vol. 51, no. 3, pp. 425-434, May 2002.

[8] J. C. S. Santos Filho, M. D. Yacoub, and G. Fraidenraich, "A simple accurate method for generating autocorrelated Nakagami- $m$ envelope sequences," IEEE Commun. Lett., vol. 11, no. 3, Mar. 2007. 
[9] E. L. Lehmann and H. J. M. D’Abrera, Nonparametrics: Statistical Methods Based on Ranks. McGraw-Hill, 1975.

[10] K. E. Baddour and N. C. Beaulieu, "Autoregressive modeling for fading channel simulation," IEEE Trans. Wireless Commun., vol. 4, no. 4, pp. 1650-1662, July 2005.

(a)

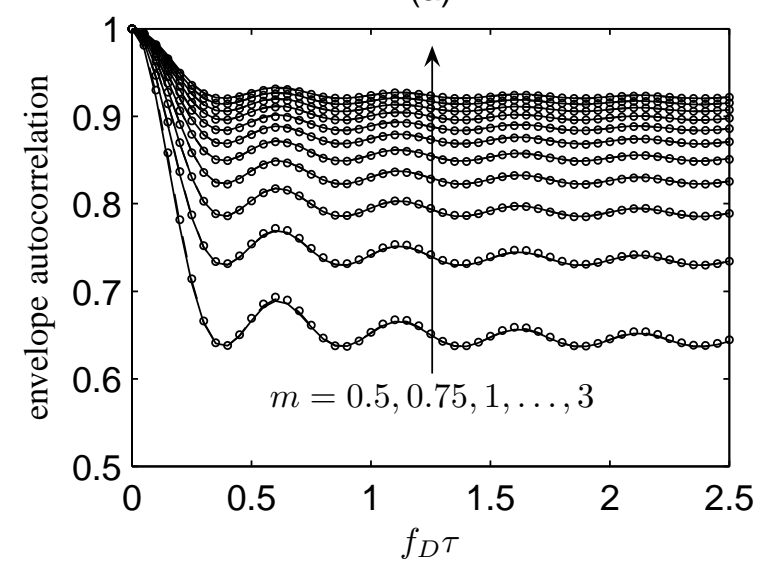

(c)

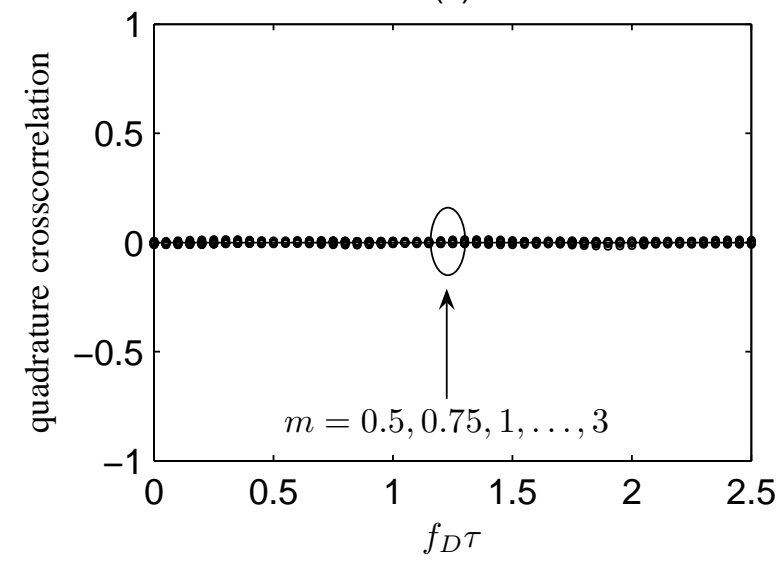

(b)

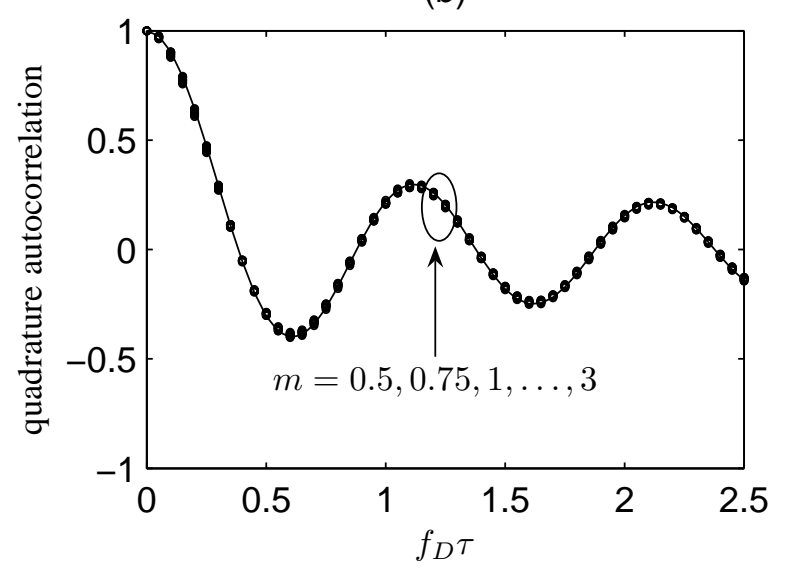

(d)

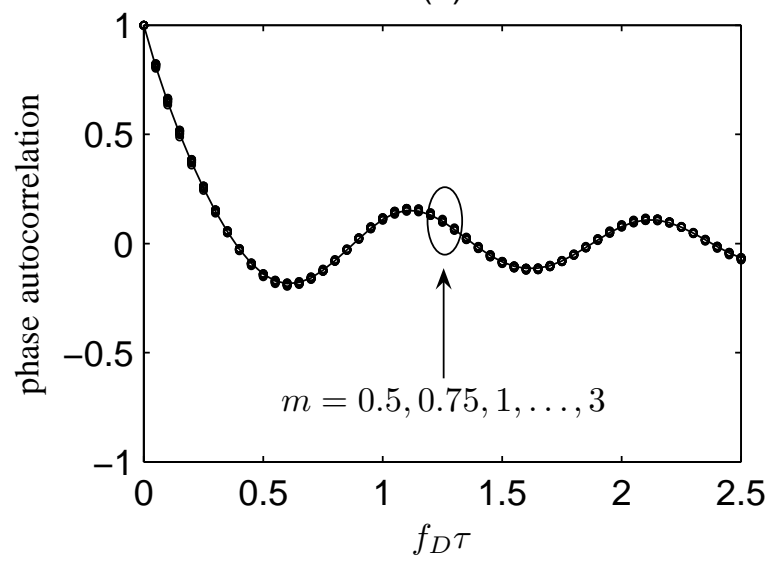

Fig. 2. Normalized auto- and crosscorrelation functions for an isotropic Nakagami channel, $\kappa=0$. (a) exact: solid; approximation: dash; simulated: circle. (b,c,d) approximation: solid; simulated: circle. 
(a)

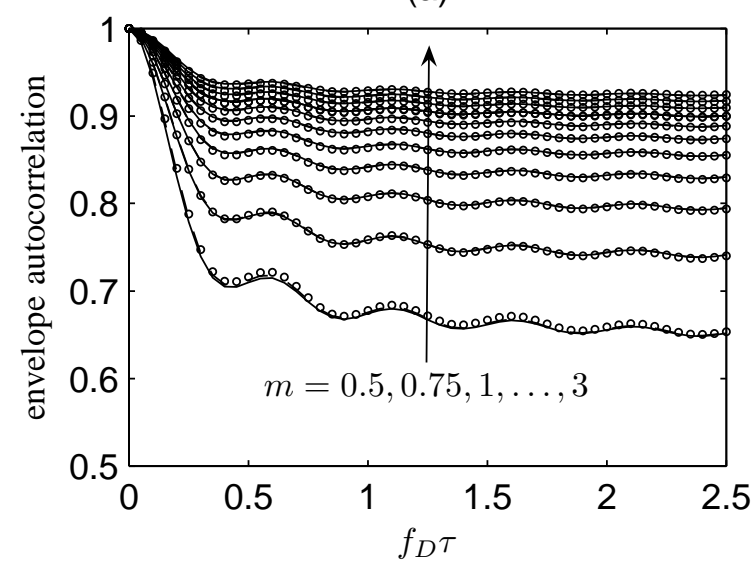

(c)

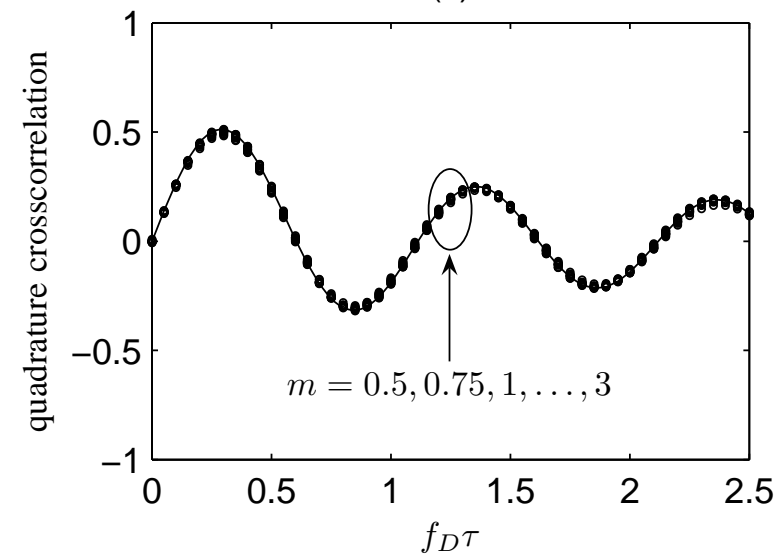

(b)

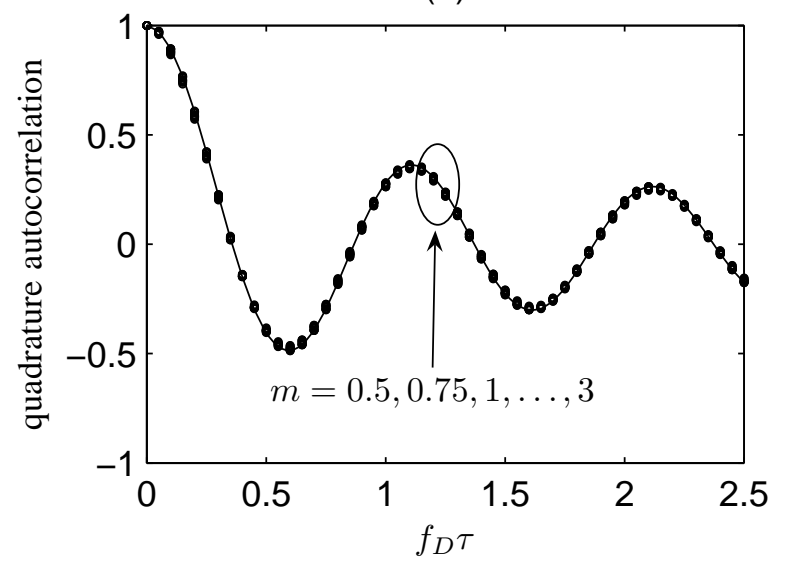

(d)

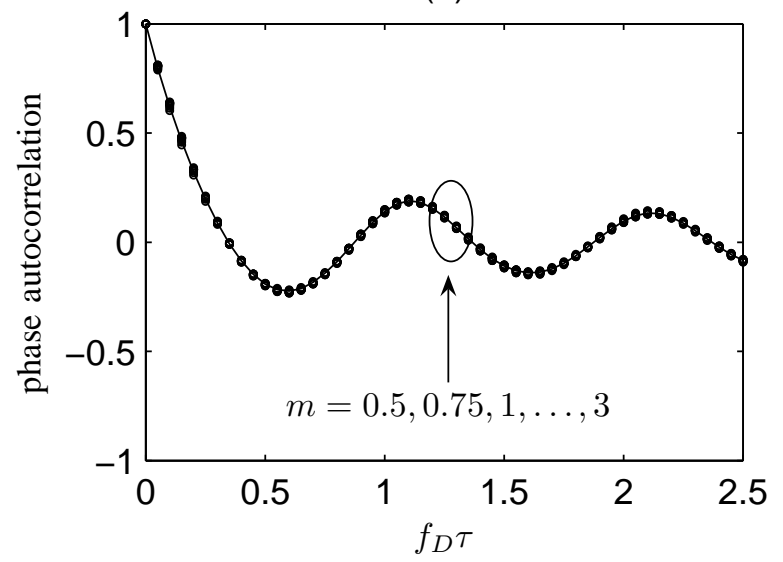

Fig. 3. Normalized auto- and crosscorrelation functions for a nonisotropic Nakagami channel, $\kappa=1$. (a) exact: solid; approximation: dash; simulated: circle. (b,c,d) approximation: solid; simulated: circle. 